Pacific Journal of Mathematics

WEAK LOCAL SUPPORTABILITY AND APPLICATIONS TO 


\title{
WEAK LOCAL SUPPORTABILITY AND APPLICATIONS TO APPROXIMATION
}

\author{
J. M. BoRWEIN
}

\begin{abstract}
Perturbed optimization problems are studied using a weaker notion of local supportability than that developed by Ekeland and Lebourg. This weakening allows for a more comprehensive treatment of such problems. In particular we prove that nearest points exist densely for closed relatively weakly compact sets in spaces with locally uniformly convex norms and provide a simplified proof in this framework that a normed space with a Fréchet norm is an Asplund space.
\end{abstract}

This paper introduces a notion of a local subgradient for a lower semicontinuous function on a Banach space. This subgradient is required to satisfy a uniformity condition on a given bounded set in the space. The first section establishes the existence of such subgradients in weakly compactly generated Banach spaces. The following sections consist of applications of this result. Section two discusses generic differentiability of convex functions and contains a simple unified proof that spaces with Fréchet norms are Asplund spaces and weakly compactly generated spaces are weak Asplund spaces. In section three general perturbed optimization problems and the existence of farthest points are discussed. Section four shows, essentially, that any relatively weakly compact set $C$ in a Banach space with a locally uniformly convex norm possesses a generic set of points with nearest points in $C$. This extends a known result for reflexive spaces.

Recently Ekeland and Lebourg [11] have introduced the notion of a local $\varepsilon$-support for a function and have profitably applied this to the study of perturbed optimization problems (including nearest and farthest points) and generic Fréchet differentiability. Rainwater [16] has provided a self-contained proof of this last result for convex functions which was deduced in [11] from more general perturbational theorems. Subsequently Lau [13] has applied the $\varepsilon$-supports to establish the existence of dense nearest points for any closed bounded set in any locally uniformly convex reflexive space. Since the results on approximation are deduced from the existence of appropriate Fréchet derivatives they are unavailable for application in more general spaces and hence do not provide best possible results (for nearest and farthest points, particularly). In this paper we introduce a more general notion of local support, examine the impli- 
cations for generic Gateaux differentiability and apply it to various approximation problems. Having made the initial adjustments many of our proofs mirror those in [11] and these propositions will be given with a minimum of proof. We therefore attempt to stay close to the notation of [11].

1. $K$-uniform local $\varepsilon$-supports. Let $V$ be a Banach space and $V^{*}$ its topological dual and let $(\cdot, \cdot)$ denote the canonical bilinear form on $V \times V^{*}$. Let $\|\cdot\|_{*}$ denote the dual norm to $\|\cdot\|$. Let $F$ : $V \rightarrow R \cup\{+\infty\}$. The effective domain of $F$, dom $F$, is the set of points at which $F$ is finite.

Let $K$ be an arbitrary closed, bounded set in $V$.

Definition 1.1. A continuous linear functional $u^{*} \in V^{*}$ is $K$ uniformly locally $\varepsilon$-supporting to $F$ at $u$ iff $F(u)<\infty$ and there exists $\eta>0$ such that

$$
\begin{gathered}
\lim _{t \rightarrow 0^{+}} \frac{F(u+t y)-F(u)}{t} \geqq\left(u^{*}, y\right)-\varepsilon \quad \forall y \in V \\
\frac{F(u+t k)-F(u)}{t} \geqq\left(u^{*}, k\right)-\varepsilon \quad \forall k \in K, 0<t<\eta .
\end{gathered}
$$

The set of all such $u^{*}$ will be denoted $S_{K, \varepsilon} F(u)$ and will be called the $K$ - $\varepsilon$-support of $F$ at $u$. If it is nonempty, $F$ is said to be locally $\varepsilon$-supported uniformly on $K$. The symbol $N_{K, \varepsilon}(F)$ will denote those $u$ for which $S_{K, \varepsilon} F(u)$ is nonempty.

REMARKS 1.2. If $K$ is empty, $u^{*}$ is an approximation to a Gateaux derivative. If $K$ is the unit ball $B$, the definition reduces to Ekeland and Lebourg's notion of $\varepsilon$-supportability. We shall be mainly interested in the case in which $K$ is (relatively) weakly compact.

These $K$ - $\varepsilon$-supports have analoguous properties to those of $\varepsilon$ supports. In particular $S_{K, \varepsilon} F(u)$ is a convex set which decreases in size as $\varepsilon$ decreases or $K$ increases and satisfies

$$
u^{*} \in \bigcap_{\varepsilon>0} S_{K, \varepsilon} F(u) \cap-S_{K, \varepsilon}(-F(u)) \Longleftrightarrow u^{*}=F_{K}^{\prime}(u) .
$$

(By $F_{K}^{\prime}(u)$ we will mean a Gateaux derivative which is approached uniformly for directions in $K$; we will say $F$ is $K$-smooth.)

Corresponding to the hypothesis $(H)$ in $[11]$ we need: 
There exists on $V$ a nonnegative nonzero continuous func$(H(K))$ tion $g$ of bounded support which is K-smooth whenever it is nonzero.

Again, by translation and homotopy, $g$ may be presumed nonzero at any given point and zero outside as small a ball as needed.

THEOREM 1.2. If $V$ satisfies $H(K)$, then for every lower semicontinuous $F, N_{K, \varepsilon}(F)$ is dense in dom $F$.

Proof. We proceed as in [11]. Pick $u_{0} \in \operatorname{dom} F$ and a small neighborhood $N$ of $u_{0}$ on which $F$ is bounded below. We now take $g$ to be nonzero at $u_{0}$ and zero outside an arbitrarily small neighborhood $N_{0} \subset N$ of $u_{0}$. Let

$$
G(u)=F(u)+h(u) ; h(u)=\frac{1}{g}(u) .
$$

$G$ is lower semicontinuous, bounded from below and hence, by Theorem 1.1 of [10], there is some point $u_{\varepsilon}$ such that

$$
G(u)-G\left(u_{\varepsilon}\right) \geqq-\frac{1}{2} \varepsilon\left\|u-u_{\varepsilon}\right\| \quad \forall u \in V .
$$

Since $g$ is $K$-smooth and $u_{\varepsilon} \in \operatorname{dom} G \subset \operatorname{dom} h,-h$ is $K$-smooth. Let $u^{*}=-h_{K}^{\prime}\left(u_{\varepsilon}\right)$. One easily verifies from (1.5) that $0 \in S_{K, \varepsilon / 2} G\left(u_{\varepsilon}^{*}\right)$ so that, by (1.3),

$$
u^{*} \in S_{K, \varepsilon / 2} G\left(u_{\varepsilon}\right)+S_{K, \varepsilon / 2}\left(-h\left(u_{\varepsilon}\right)\right) \subset S_{K, \varepsilon} F\left(u_{\varepsilon}\right) .
$$

Moreover, $u_{\varepsilon} \in N_{0}$, which establishes the density claim.

To give this substance we note that any $V$ which has an equivalent $K$-smooth norm has $H(K)$. Again as in [11], one takes the composition of the norm with a $C_{1}$ function of bounded support. Less trivially we have the following proposition.

Proposition 1.3. Let $V^{*}$ have an equivalent strictly convex dual norm, $\|\cdot\|_{*}$ and let $W$ be any weakly compact set. Then $V$ has an equivalent $W$-smooth norm and hence has $H(W)$.

Proof. Let $V_{0}$ be the subspace which is the closed linear span of $W\left(V_{0}=\overline{\mathrm{sp}} W\right)$. By the results in [6, pg. 161] (on factorization of weakly compact operators), these is a reflexive space $(Y,\||\cdot|\|)$ with unit ball $B(Y)$ and a continuous linear operator $T: Y \rightarrow V_{0}$ such that $W \subset T(B(Y))$. Let $\bar{T}$ denote the mapping $T$ considered as mapping $Y$ into $V$ and define a new norm $|\cdot|_{*}$ on $V^{*}$ by 


$$
|f|_{*}^{2}=\|f\|_{*}^{2}+\left\||| \bar{T}^{*} f\right\|_{*}^{2} \text {. }
$$

By the renorming theorem of Troyanksi [19] we may presume that $\left.\left|\|\mid\| \|_{*}\right.$ is locally uniformly convex. Note that $| \cdot\right|_{*}$ is equivalent to $\|\cdot\|_{*}$ as $T^{*}$ is continuous and is a dual norm since $T^{*}$ is an adjoint mapping. Thus $|\cdot|_{*}$ induces an equivalent norm $|\cdot|$ on $V$. We show that $|\cdot|$ is $W$-smooth. Notice first that $|\cdot|$ is smooth since $|\cdot|_{*}$ is strictly convex. Let $u_{0} \in V$, and let $u_{n}$ converge to $u_{0}$ in norm. Since $|\cdot|$ is smooth the support functionals $f_{u_{n}}$ converge weak* to $f_{u_{0}}$. Thus $\left|f_{u_{n}}+f\right|_{*} \rightarrow 2$. It follows from (1.6) that $\||| \bar{T} f_{u_{n}}+\bar{T} f_{u_{0}}||_{*}^{2}-2\left(|| \bar{T} f_{u_{n}}||_{*}^{2}+||\left|\bar{T} f_{u_{0}}\right| \|_{*}^{2}\right)$ converges to zero with $n$. Since $\|\cdot\|_{*}$ is locally uniformly convex, $\left\|\bar{T} f_{u_{n}}-f_{u_{0}}\right\| \|_{*} \rightarrow 0$. In other words, given $\varepsilon>0$, there is some $n_{0}$ such that for $n \geqq n_{0}$

$$
\sup _{b \in B(Y)}\left(\bar{T}_{*}\left(f_{u_{n}}-f_{u_{0}}\right), b\right)=\sup _{b \in B(Y)}\left(f_{u_{n}}-f_{u_{0}}, T b\right) \leqq \varepsilon .
$$

Since $W \subset T(B(Y))$, we have

$$
\sup _{w \in W}\left(f_{u_{n}}-f_{u_{0}}, w\right) \longrightarrow 0 \text { as } n \longrightarrow \infty \text {. }
$$

The standard equation [6, pg. 2]

$$
\frac{f_{u}}{|u|}(y) \leqq \frac{|u+t y|-|u|}{t} \leqq \frac{f_{u+t y}(y)}{|u+t y|}
$$

which holds for any $t>0$ and any nonzero $y$ and (1.7) now show that $|u+t k|-|u| / t$ converges to $f_{u}(k) /|u|$ uniformly for $k$ in $W$. Thus $|\cdot|$ is $W$-smooth.

In particular, when $V$ is weakly compactly generated the result holds and $W$ can be chosen to be densely spanning and absolutely convex. In this case the norm of (1.6) can be said to be "almost" Fréchet. This is the important case for applications. The sequence space $l_{1}(N)$ is an example of a space without property $(H)$ to which the proposition applies. It will be convenient from now on to denote

$$
\left\|u^{*}\right\|_{K}=\sup _{k \in K}\left(u^{*}, k\right)
$$

and to consider $\|\cdot\|_{K}$ as the norm on $C_{b}(K)$, the space of bounded continuous functions on $K$ endowed with the norm topology.

2. An application to $K$-Asplund spaces. We will say $V$ is $K$-Asplund if given any lower semicontinuous convex function $f: V \rightarrow$ $R \cup\{+\infty\}, f$ is $K$-smooth on a dense $G_{\delta}$ subset of $(\operatorname{dom} f)^{\circ}$. Thus for $K=B(\phi)$ we have the classical notion of a strong (weak) Asplund space [2], [5]. We introduce the set $M_{K, \varepsilon}(f)$ by 


$$
\begin{aligned}
& M_{K, \varepsilon}(f) \\
\text { (2.1) } & =\left\{u: \exists \delta>0 \text { with } \sup _{\substack{k \in K \\
0<t<\delta}} \frac{f(u+t k)+f(u-t k)-2 f(u)}{t}<\varepsilon\right\} .
\end{aligned}
$$

When $K$ is the unit ball and $f$ is a norm, Sullivan [18] has studied such sets and called $f \varepsilon$-approximately Fréchet. When $K=$ $\phi, M_{K, \varepsilon}(f)$ is just $\operatorname{dom} f$.

Proposition 2.1. Let $K$ be a symmetric set. Then

(i) $N_{K, \delta}(f) \subset M_{K, \varepsilon}(f)$ whenever $2 \delta<\varepsilon$.

(ii) If $f$ is convex and lower semicontinuous $M_{K, \varepsilon}(f)$ is open in $(\operatorname{dom} f)^{\circ}$.

(iii) If $u \in \bigcap_{\varepsilon>0} M_{K, \varepsilon}(f) \cap(\operatorname{dom} f)^{\circ}$ and $K$ has closed span $V$ then $f$ is $K$-smooth at $u$.

Proof. (i ) follows on applying (1.2) to $-f$ for $k$ and $-k$ and then adding the resulting expressions.

(ii) Since $f$ is convex it is in fact locally Lipschitz on (dom $f)^{\circ}$ [5]. Also in this case

$$
\begin{aligned}
M_{K, \varepsilon}(f) & =\bigcup_{n}\left\{u: \sup _{k \in K} \frac{f\left(u+\frac{1}{n} k\right)+f\left(u-\frac{1}{n} k\right)-2 f(u)}{\frac{1}{n}}<\varepsilon\right\} \\
& =\bigcup_{n} M_{n} .
\end{aligned}
$$

Suppose $u \in M_{n}$ and $\sup _{k \in K} f(u+t k)+f(u-t k)-2 f(u) / t<\varepsilon-\delta$. Now pick $\eta$ such that $\left\|u-u_{1}\right\|,\left\|u-u_{2}\right\| \leqq 2 \eta$ implies $\| f\left(u_{1}\right)-$ $f\left(u_{2}\right)\|\leqq L\| u_{1}-u_{2} \|$. Then if $m \geqq \max (1 / \eta, n)$ and if $\|v-u\| \leqq \eta$ it follows that $u \pm(1 / m) k, v \pm(1 / m) k, v$ are all within $2 \eta$ of $u$. Finally, when $\|v-u\| \leqq \delta / 4 L m$

$$
\begin{gathered}
\left|\frac{f\left(v+\frac{1}{m} k\right)+f\left(v-\frac{1}{m} k\right)-2 f(v)}{\frac{1}{m}}-\frac{f\left(u+\frac{1}{m} k\right)+f\left(u-\frac{1}{m} k\right)-2 f(u) \mid}{\frac{1}{m}}\right| \\
\leqq \frac{4 L\|v-u\|}{\frac{1}{m}} \leqq \delta .
\end{gathered}
$$

Since $f(u+(1 / m) k)+f(u-(1 / m) k)-2 f(u) / 1 / m$ is a decreasing function of $m$, it follows that $v \in M_{m}$ and $M_{K, \varepsilon}(f)$ is open in $(\operatorname{dom} f)^{\circ}$.

(iii) Since $f$ is convex, $d^{+} f(u ; h)=\lim _{t \rightarrow 0^{+}} f(u+t h)-f(u) / t$ exists and is finite at $u$. Since $u \in \bigcap_{\varepsilon>0} M_{\varepsilon, K}(f)$, we see that $d^{+} f(u ; k)=$ $-d^{+} f(u ;-k)$ for all $k \in K$. Since $d^{+} f(u ; k)$ is continuous in $k$ and 
$K$ is generating, we must have $d^{+} f(u ; h)=-d^{+} f(u ;-h)$ identically in $h$ which implies that $f$ is Gateaux differentiable with derivative $d^{+} f(u ; \cdot)$. Let us call this derivative $u^{*}$. Since for $t>0$ and $k \in$ $K$ we have

$$
\frac{f(u+t k)-f(u)}{t} \geqq\left(u^{*}, k\right) \geqq \frac{f(u-t k)-f(u)}{-t},
$$

and as $u \in \bigcap_{\varepsilon>0} M_{K, \varepsilon}(f)$, we must have for each $\varepsilon>0$, some $\delta$ with

$$
\left|\frac{f(u+t k)-f(u)}{t}-\left(u^{*}, k\right)\right|<\varepsilon \forall k \in K, 0<t<\delta .
$$

Thus $f$ is $K$-smooth at $u$.

We will say that $K$ is generating for $V$ if the closed span of $K$ is $V$.

THEOREM 2.2. If $V$ has property $H(K)$ for some symmetric spanning set $K, V$ is $K$-Asplund. In particular every space with $(H)$ is a strong-Asplund space and every weakly compactly generated space is $K$-Asplund for any generating weakly compact set $K$.

Proof. On collecting the parts of Proposition 2.1 together it suffices to observe that by Theorem 1.2, $N_{K, \varepsilon}(-f)$ is dense in (dom $f)^{\circ}$ for any l.s.c. convex function $f$ (an examination of the proof of Theorem 1.2 shows that it does not matter that $-f(u)$ may be $-\infty$ for $u$ not in $\left.(\operatorname{dom} f)^{\circ}\right)$. Thus by Baire's theorem $\bigcap_{\varepsilon>0} M_{K, \varepsilon}(-f)$ is a dense $G_{\delta}$ set in $(\operatorname{dom} f)^{\circ}$ on which $f$ is $K$-smooth.

This proof method has unfortunately no hope of dealing with the general question of when a space is weak-Asplund, but it does provide a self-contained proof of several different results at the same time. We also remark that if $f$ is a norm and $N_{B, o}(f)=V$ for some $\delta<1 / 2$ then $V$ is a strong Asplund space. This is a trivial consequence of the fact that by Corollary 2.1 (i), $M_{B, s}(\|\cdot\|)=V$ for some $\varepsilon<1$ which Sullivan [18] has shown implies $V$ is an Asplund space.

3. Perturbed optimization. We now examine the substance of $\S 2$ of [11] for our more general local supports. Throughout the section we suppose $K$ is convex, symmetric and generating. Let $X$ be a set and $f: V \times X \rightarrow R$. Let

$$
F(u)=\inf _{x \in X} f(u, x)>-\infty \quad \forall u \in V .
$$


Let $A_{\theta}=\{x \in X: f(u, x) \leqq F(u)+\theta\}$.

Proposition 3.1. Assume that

$$
F \text { is K-uniformly locally e-supported to } u
$$

and that there exist $\theta>0, \eta>0$ such that

(3.3) $\forall x \in A_{\theta}, v \rightarrow f(v, x)$ is Frechet-differentiable at $v$ for

$$
\|v-u\| \leqq \eta
$$

(3.4) $\quad\left\{v \rightarrow f_{v}^{\prime}(v, x): x \in A_{\theta}\right\}$ is equicontinuous at $u$ in $V^{*}$.

Then there exists $\eta_{1}, \theta_{1}>0$ such that

$$
\text { diameter }\left\{f_{v}^{\prime}(v, x):\|v-u\| \leqq \eta_{1}, x \in A_{\theta_{1}}\right\} \leqq \varepsilon / 8 \text { in } C_{b}(K) \text {. }
$$

Proof. The proof is more or less line for line as that of Proposition 2.2 of [11]. We note that since we are only interested in directions in $K$ and distances in $C(K)$ metric we need only assume that $K-\varepsilon$ supports exist.

Let

$$
A_{u, K}^{*}(\eta, \theta)=\left\{f_{v}^{\prime}(v, x):\|v-u\| \leqq \eta, f(u, x) \leqq F(u)+\theta\right\}
$$

and let $T_{\varepsilon}(K)$ be defined by

$$
u \in T_{\varepsilon}(K) \Longleftrightarrow \exists \eta, \theta>0, C_{b}(K) \text {-diameter } A_{u, K}^{*}(\eta, \theta) \leqq \varepsilon .
$$

Proposition 3.2. Assume $u \in T_{\varepsilon}(K)$ and that there exist $\alpha, \beta>$ 0 such that

$$
\begin{aligned}
& \left\{f_{v}^{\prime}(v, x):\|v-u\| \leqq \alpha, f(v, x) \leqq F(u)+\beta\right\} \text { is norm bounded } \\
& \text { in } V^{*} .
\end{aligned}
$$

Then $T_{s}(K)$ is a norm neighborhood of $u$.

Proof. This is now exactly as Proposition 2.3 of [11].

Proposition 3.3. If (3.3), (3.4) and (3.8) hold $F$ is locally Lipshitzian at $u$. Moreover, if the filter $G_{u}(K)$ generated by $\left\{A_{u, K}^{*}(\theta, \eta): \theta ; \eta>0\right\}$ converges to $u^{*}$ in $C_{b}(K), F$ is $K$-smooth at $u$ with $F_{K}^{\prime}(u)=u^{*}$.

Proof. Again the only real difference from Proposition 2.4 of [11] is that we are only concerned with directions in $K$ and the topology in $C_{b}(K)$ rather in $C_{b}(B)$. We derive as in [11] that $F$ is 
Lipschitz locally at $u$ and that

$$
\lim _{t \rightarrow 0^{+}} \frac{F(u+t k)-F(u)}{t}-\left(u^{*}, k\right)=0
$$

uniformly for $k \in K$. This in conjunction with $\overline{\mathrm{U}_{n \in N} n K}=V$ and the locally Lipschitzian nature of $F$ implies that $F^{\prime}(u)$ exists as a Gateaux derivative. Hence $F$ is $K$-smooth.

Thus, collecting propositions yields:

THEOREM 3.3. Suppose that $V$ satisfies $H(K)$ for some symmetric, convex generating set $K$ and that for some open set $\Omega \subset V$ every point of $u$ satisfies (3.3), (3.4), (3.8).

Then $F$ is locally Lipshitzian on $\Omega$. Moreover, there is a dense $G_{\delta}$ subset $T$ of $\Omega$ such that

$$
\text { the filter } G_{u}(K) \text { converges in } C_{b}(K) \text {, }
$$

and

$$
\begin{aligned}
& F \text { is } K \text {-smooth with } F_{K}^{\prime}(u) \text { the limit of } G_{u}(K) \text {; also } F_{K}^{\prime} \\
& \text { is continuous from } T \text { to } C_{b}(K) \text {. }
\end{aligned}
$$

Proof. By Proposition $3.3 F$ is locally Lipschitz on $\Omega$. By Theorem 1.2, for $\varepsilon>0, N_{K, \varepsilon}(F)$ is dense in $\Omega$. By Propositions 3.1 and 3.2, $T_{\varepsilon}(K)$ is dense and open in $\Omega$. By Baire's theorem (on $\Omega$ ) $T=\bigcap_{n \in N} T_{1 / n}(K)$ is a dense $G_{\delta}$ set in $\Omega$.

Let $u \in T$. For each $n$ in $N, G_{u}$ contains a member of $C_{b}(K)$ diameter less than $1 / n$. By (3.3), (3.8) the filter members eventually are uniformly bounded in the norm topology on $V^{*}$. Since $C_{b}(K)$ is complete the filter converges to some element $g$ in $C_{b}(K)$. Since $\overline{U n K}=V$ and the filter members are eventually uniformly bounded in $V^{*}$, the filter converges pointwise to a functional $u^{*}$ in $V^{*}$ which extends $g$. By Proposition 3.3 this limit is $F_{K}^{\prime}(u)$.

Continuity in $C_{b}(K)$ now follows as in Theorem 2.5 of [11].

COROLLARY 3.4. If $V$ is weakly compactly generated, the conclusions hold for any weakly compact generating set. If $V$ has property $(H)$ the conclusions hold in the norm topology in $V^{*}$.

We now consider general convex suprema as an application. Let $f(u, x)$ be a family of lower semicontinuous convex functions. Using conjugate convex functions $\left(h^{*}\left(u^{*}\right)=\sup _{u \in V}\left(u^{*}, u\right)-h(u)\right)$ we write (using $h^{* *}=h$ ) 


$$
F(u)=\sup _{x \in X} f(u, x)=\sup _{u^{*} \in V^{*}, x \in X}\left(u^{*}, u\right)-f^{*}\left(u^{*}, x\right)<\infty .
$$

THEOREM 3.5. Suppose $V$ is weakly compactly generated by $W$. Suppose that $f: V \times X \rightarrow R$ is continuous and convex in $u$ for each $x$ and that for each $u$ in $V$ there is some $M>0$ with

$$
f(u, x) \leqq M \text { for } x \in X .
$$

There is a dense $G_{\delta}$ set $T$ in $V$ such that $F_{w}^{\prime}(u)$ exists for $u$ in $T$ and such that

$$
\left(u_{n}^{*}, u\right)-f^{*}\left(u_{n}^{*}, x_{n}\right) \longrightarrow F(u) \Longrightarrow\left\|u_{n}^{*}-F_{W}^{\prime}(u)\right\|_{W} \longrightarrow 0 \text {. }
$$

Proof. We may assume $W$ is symmetric and convex. It suffices to verify that the suprema version of (3.5), (3.6), (3.8) hold for the functions $\left(u^{*}, u\right)-f^{*}\left(u^{*}, x\right)$. Since each of these has derivative $u^{*}$ with respect to $u$, it suffices to show that for some $m>0$, there exists $c>0$ such that

$$
\begin{gathered}
\|u-v\| \leqq \eta \text { and }\left(u^{*}, v\right)-f\left(u^{*}, x\right) \geqq F(u)-1 \\
\Longrightarrow\left\|u^{*}\right\| \leqq c .
\end{gathered}
$$

By hypothesis $F$ is continuous (being finite and lower semicontinuous) so there exists $\eta>0$ with $f(v, x) \leqq F(u)+1$ when $\| v-$ $u \| \leqq 2 \eta$. For these $v$ we have, therefore,

$$
f^{*}\left(u^{*}, x\right) \leqq 2 \eta\left\|u^{*}\right\|_{*}+\left(u^{*}, v\right)-F(u)+1
$$

and so if $f^{*}\left(u^{*}, x\right) \leqq\left(u^{*}, v\right)+F(u)+1$ and $\|u-v\| \leqq \eta$

$$
2 \geqq 2 \eta\left\|u^{*}\right\|_{*}+\left(u^{*}, v-u\right) \geqq \eta\left\|u^{*}\right\|_{*} \text {. }
$$

The remaining conclusion is now a direct application of (3.10).

CoRollary 3.6. Let $f(u, x)=f(u-x)+g(x)$ where $X$ is a weakly compact subset of $V, f$ is bounded on bounded sets and convex and $g$ is weally upper semicontinuous. (i) Then there is a dense $G_{\delta}$ set $T$ in $V$ such that for $u \in T, F_{W}^{\prime}(u)$ exists and

$$
F(u)=\max _{x \in X} f(x-u)+g(x) .
$$

(ii) If, in addition, $f$ is a locally uniformly convex function then it suffices that $X$ is relatively weakly compact and $g$ is norm upper semicontinuous and bounded.

Proof. (i) We may suppose that $X \subset W$. Since $X$ is bounded and $f$ is bounded on bounded sets (3.11) holds. Let $x_{n} \in X, u_{n}^{*} \in V^{*}$ 
be a minimizing sequence. We may assume that $x_{n}$ converges weakly to $x_{0}$. On using $(3.12)$ we see that $\left(u_{n}^{*}, x_{n}\right)$ converges to $\left(F_{W}^{\prime}(u), x_{0}\right)$. Moreover,

$$
\left(u^{*}, u\right)-f^{*}\left(u^{*}, x\right)=\left(u^{*}, u-x\right)+g(x)-f^{*}\left(u^{*}\right) .
$$

As $u_{n}^{*}$ also converges weak ${ }^{*}$ to $u_{0}^{*}=F_{W}^{\prime}(u)$ and $f^{*}$, being a conjugate is weak* lower semicontinuous, $f^{*}\left(u_{0}^{*}\right) \leqq \lim f^{*}\left(u_{n}^{*}\right)$. Also $g\left(x_{0}\right) \geqq \varlimsup \lim g\left(x_{n}\right)$. Thus

$$
\begin{aligned}
F(u) & \geqq f\left(u-x_{0}\right)+g\left(x_{0}\right) \geqq\left(u_{0}^{*}, u\right)-f^{*}\left(u_{0}^{*}, x_{0}\right) \\
& \geqq \lim _{n}\left(u_{0}^{*}, u-x_{n}\right)+g\left(x_{n}\right)-f^{*}\left(u_{0}^{*}\right) \\
& \geqq \lim _{n}\left(u_{n}^{*}, u-x_{n}\right)+g\left(x_{n}\right)-f^{*}\left(u_{n}^{*}\right) \\
& =F(u) .
\end{aligned}
$$

(ii) Suppose $X$ is relatively weakly compact. We may apply the previous considerations to $C=\overline{\mathrm{co}} X$ and deduce that some sequence $x_{n}$ in $X$ exists with $\lim g\left(x_{n}\right)=g_{0}$ and

$$
F(u)=\lim _{n}\left(u_{0}^{*}, u-x_{n}\right)-f^{*}\left(u_{0}^{*}\right)+g\left(x_{n}\right) \leqq f\left(u-x_{0}\right)+g_{0} .
$$

Since $f\left(u-x_{n}\right)+g\left(x_{n}\right) \leqq F(u)$ and $f$ is weakly lower semicontinuous we must have

$$
f\left(u-x_{n}\right) \longrightarrow f\left(u-x_{0}\right) ; x_{n} \longrightarrow x_{0} \text { weakly. }
$$

(Here we have used the fact that $\sup _{x \in C} f(u-x)=\sup _{x \in X} f(u-x)$ for any lower semicontinuous convex function.) It follows from (3.15) and (3.16) that

$$
\lim _{n} f\left(\frac{u-x_{n}}{2}+\frac{u-x_{0}}{2}\right) \geqq \lim _{n}\left(u_{0}^{*}, u-x_{n}\right)-f^{*}\left(u_{0}^{*}\right)=f\left(u-x_{0}\right) .
$$

Thus

(3.17) $\lim _{n} f\left(\frac{u-x_{n}}{2}+\frac{u-x_{0}}{2}\right)-\frac{1}{2}\left[f\left(u-x_{n}\right)+f\left(u-x_{0}\right)\right] \longrightarrow 0$.

To say that $f$ is locally uniformly convex is to say that (3.17) implies $u-x_{n} / 2$ converges in norm to $u-x_{0} / 2$. Thus $x_{n} \rightarrow x_{0}$ in norm and $x_{0} \in X$. This means that $g\left(x_{0}\right) \geqq g_{0}$ and (3.15) completes the proof.

REMARKS 3.7. (i) If we let $X$ be singleton in Theorem 3.5 we recover one of the main results of Theorem 2.2.

(ii) In Corollary 3.6 (ii) one may also consider functions for which (3.16) implies $\left\{x_{n}\right\}$ converges in norm and proceed without 
(3.17). This is done in [3] for reflexive spaces with $f=\omega(\|\cdot\|)$ where $\omega$ is a convex continuous strictly increasing function and $\|\cdot\|$ has property $(\kappa)$ (see $\S 4$ ).

(iii) The case of Corollary 3.6 (ii) in which $g=0$ and $f$ is the square of a norm which is locally uniformly convex is proved by Lau in [14]. He has no requirement that the space be weakly compactly generated. However, this requirement can be weakened if one wishes to dispense with the differentiability of $F$. Moreover, in applications this is little liability since $\overline{\operatorname{span}} X$ is weakly compactly generated. Since there is always an equivalent locally uniformly convex norm for $\overline{\operatorname{span}} X$ [6] and since any farthest point in this norm for $u$ in $X$ necessarily is a strongly exposed point of $X$, one can then proceed as in Lau's papers [15], [12] to derive Lindenstrauss-Troyanski's original result [19] that every weakly compact convex set is the closed convex hull of its strongly exposed points (see the remark at the bottom of page 209 of [11]).

COROLlaRY 3.8. Let $V$ be a weakly compactly generated space (generated by $W$ ) and let $C^{*}$ be a weak*-compact subset of $V^{*}$. There is a dense $G_{\delta}$ set $T \subset V$ such that if $u \in T$ there is some $c^{*} \in$ $C^{*}$ with

$$
\left(c^{*}, u\right)=\max _{u^{*} \in O^{*}}\left(u^{*}, u\right)
$$

and

$$
\left(c_{n}^{*}-c^{*}, u\right) \longrightarrow 0, c_{n}^{*} \in C^{*} \longrightarrow\left\|c_{n}^{*}-c^{*}\right\|_{W} \longrightarrow 0 .
$$

In particular, $C^{*}$ is the weak* closed convex hull of these points satisfying (3.19).

Proof. Theorem 3.5 with $X=C^{*}, x=u^{*}, f\left(u, u^{*}\right)=\left(u^{*}, u\right)$ implies (3.18) and (3.19) and the final conclusion now follows from a standard separation argument.

Since $W$ is spanning any point satisfying (3.19) is at least weak*-exposed and in fact a good deal more. In reflexive spaces they are strongly exposed points. It would be interesting to study these $W$-weak* strongly exposed points more carefully, particularly in duality with $W$-Asplund spaces.

REMARK 3.9. It suffices for the results of this section that (3.3) be replaced by

$$
\forall x \in A_{\theta}, v \longrightarrow f(v, x) \text { is } K \text {-smooth at } v \text { for }\|v-u\| \leqq \eta .
$$


4. Nearest points. As a final application of $K-\varepsilon$-support points we indicate an adaptation of Lau's proof method in [13] which establishes the following result. We say a norm has property $(\kappa)$ if whenever $\left\|x_{n}\right\|$ converges to $\|x\|$ and $x_{n}$ converges weakly to $x$ we have $x_{n}$ converges in norm to $x$. Locally uniformly convex spaces and $l_{1}(S)$ on any set $S$ are among the spaces with this property.

Theorem 4.1. Suppose $V$ has property ( $\kappa$ ) and $V^{*}$ has an equivalent strictly convex dual norm. Let $K$ be a relatively weakly compact, norm closed subset of $V$. There exists in $V$ a dense $G_{\delta}$ set $T$ such that each point $u$ in $T$ has a nearest point in $K$. That is: there exists $k_{0}$ in $K$ with

$$
\left\|u-k_{0}\right\|=\inf _{k \in K}\|u-k\| .
$$

Proof. Let $r(u)=\inf _{k \in K}\|u-k\|$. Let $0<\varepsilon<1$ be given and define $A_{\varepsilon}\left(\delta, u^{*}\right)$ and $A_{\varepsilon}$ by

$$
\begin{aligned}
A_{\varepsilon}\left(\delta, u^{*}\right) & =\{u \in V / K: k \in K,\|k-u\| \leqq r(u)+\delta \\
& \left.=\left(u^{*}, k-u\right) \leqq(\varepsilon-1) r(u)\right\} \\
A_{\varepsilon}= & \cup\left\{A_{\varepsilon}\left(\delta, u^{*}\right):\left|\left\|u^{*}\right\|-1\right|<\varepsilon, \delta>0\right\} .
\end{aligned}
$$

In [13], Lau shows that $A_{\varepsilon}$ is open and dense in $V / K$ whenever $V$ has property $(\mathrm{H})$. He then shows that $T=\bigcap_{n \in N} A_{1 / n}$ is the desired set possessing nearest points. Lau's proof that $A_{\varepsilon}$ is open requires no special hypotheses on $K$ or $V$ and his proof that $T$ satisfies (3.20) relies only on property $(\kappa)$ and relative weak compactness of $K$.

Lau in fact shows that, for any point $u$ in $T$ and any weakly convergent minimizing sequence $k_{n}$ in $K$ converging to $k_{0}$ in the weak closure of $K$, one has $\left\|k_{n}-u\right\|$ converging to $\left\|k_{0}-u\right\|$. It now follows from $(\kappa)$ that $k_{0}$ lies in $K$ and the infimum is attained.

It remains to show that $A_{\varepsilon}$ is dense even in absence of property $(\mathrm{H})$. We do this in the following propositions.

Proposition 4.2. Given $\varepsilon, \eta>0$ and $u_{0} \in V / K$, there exist $\bar{u}$ in $V, \bar{u}^{*}$ in $V^{*}$ and $t_{0}>0$ such that

$$
\begin{gathered}
\left\|\bar{u}-u_{0}\right\| \leqq \eta, \bar{u}^{*} \in S_{k, s} r(\bar{u}), \\
\frac{r(\bar{u}+t(k-\bar{u}))-r(\bar{u})}{t} \geqq\left(\bar{u}^{*}, k-\bar{u}\right)-\varepsilon\|k-\bar{u}\| \quad \forall k \in K, 0<t<t_{0} .
\end{gathered}
$$

Proof. An examination of (1.7) and (1.8) shows that the norm 
of (1.6) actually satisfies the additional equation:

$$
\lim _{t \rightarrow 0^{+}} \frac{|u+t(k-u)|-|u|}{t}=\frac{f_{u}(k-u)}{|u|}
$$

uniformly for $k$ in $K$. We can now produce a $K$-smooth function of bounded support satisfying the same additional uniformity. If we repeat the process of Theorem 1.2 for $r$ we derive (in addition to $\left.u^{*} \in S_{K, \varepsilon} r\left(u_{\varepsilon}\right)\right)$ that

$$
\frac{G\left(u_{\varepsilon}+t\left(k-u_{\varepsilon}\right)\right)-G\left(u_{\varepsilon}\right)}{t} \geqq-\frac{\varepsilon}{2}\left\|k-u_{\varepsilon}\right\|
$$

and, setting $d=(1 / 2) r\left(u_{0}\right)$,

$$
\frac{-h\left(u_{\varepsilon}+t\left(k-u_{\varepsilon}\right)\right)+h\left(u_{\varepsilon}\right)}{t} \geqq\left(u^{*}, k-u_{\varepsilon}\right)-\frac{\varepsilon}{2} d
$$

whenever $0<t<\delta_{1}$. If we arrange in advance for $g$ to have its support in a neighborhood of $u_{0}$ of diameter less than both $d$ and $\eta$, it follows that $\left\|u_{\varepsilon}-u_{0}\right\|<d$ and so $r\left(u_{\varepsilon}\right)>d$. Then (3.27) and (3.28) combine to give

$$
\begin{aligned}
\frac{r\left(u_{\varepsilon}+t\left(k-u_{\varepsilon}\right)\right)-r\left(u_{\varepsilon}\right)}{t} & \geqq\left(u^{*}, k-u_{\varepsilon}\right)-\frac{\varepsilon}{2}\left(d+\left\|k-u_{\varepsilon}\right\|\right) \\
& \geqq+\left(u^{*}, k-u_{\varepsilon}\right)-\varepsilon\left\|k-u_{\varepsilon}\right\|
\end{aligned}
$$

for $0<t \leqq t_{0}\left(t_{0}\right.$ depends on $K$ and $\left.u_{0}\right)$ and $k \in K$. Since $\left\|u_{\varepsilon}-u_{0}\right\| \leqq$ $\eta, u=u_{\varepsilon}$ and $\bar{u}^{*}=u^{*}$ are the desired points.

Proposition 4.3. If $u, u^{*}$ satisfy (3.23), (3.24) for $\varepsilon>0$, then $u \in A_{2 \varepsilon}$. It follows that $A_{2 \varepsilon}$ is dense in $V / K$.

Proof. Let $\delta=\left(t_{0} \varepsilon r(u)\right) / 2$ where $0<\varepsilon<1 / 2$ and $t_{0}$ are as above. Suppose that

$$
k \in K \text { and }\|k-u\| \leqq r(u)+\delta .
$$

Since $(1-t)\|k-u\| \geqq r(u+t(k-u))$, (3.30) and (3.25) combine to give

$$
(1-t)\|k-u\|-\|k-u\|+\delta \geqq t\left(u^{*}, k-u\right)-t \varepsilon\|k-u\|
$$

if $0<t \leqq t_{0}$ and $k$ satisfies (3.30). Thus, setting $t=t_{0}$,

$$
-r(u)+\frac{\delta}{t_{0}} \geqq\left(u^{*}, k-u\right)-\varepsilon\|k-u\|
$$




$$
(-1+2 \varepsilon) r(u) \geqq-r(u)+\frac{\varepsilon}{2} r(u)+\varepsilon(r(u)+\delta) \geqq\left(u^{*}, k-u\right),
$$

for $k$ satisfying (3.30). Once we have shown that $\left|1-\left\|u^{*}\right\|\right|<2 \varepsilon$ it will follow that $u \in A_{2 \varepsilon}\left(\delta, u^{*}\right) \subset A_{2 \varepsilon}$ and $u_{0} \in \bar{A}_{2 \varepsilon}$ as required. This final assertion is proven below.

Proposition 4.4. If $u^{*}$ lies in $S_{K, \varepsilon} r(u)$, then

$$
||\left|u^{*}\right||-1| \leqq \varepsilon .
$$

Proof. For any $v$ in $V$ we have by (1.1)

$$
\frac{r(u+t v)-r(u)}{t} \geqq\left(u^{*}, h\right)-\varepsilon \quad 0<t<t(v) .
$$

Setting $v=h /\|h\|, s=t /\|h\|$ we have

$$
\frac{r(u+s h)-r(u)}{s} \geqq\left(u^{*}, h\right)-\varepsilon\|h\| \quad 0<s<s(h) .
$$

Since $r$ has Lipschitz constant one (3.32) produces

$$
(1+\varepsilon)\|h\| \geqq u^{*}(h) \text { and }\left\|u^{*}\right\| \leqq 1+\varepsilon .
$$

Let $\left\|u-k_{n}\right\|-r(u)=\varepsilon_{n}$ converge to 0 . Pick $t_{n}$ with $t_{n} \| u-$ $k_{n} \|=\varepsilon_{n}^{1 / 2}$ so that

$$
\begin{aligned}
\frac{r(u)-r\left(u+t_{n}\left(k_{n}-u\right)\right)}{t_{n}\left\|k_{n}-u\right\|} & \geqq \frac{\left\|u-k_{n}\right\|-\varepsilon_{n}-\left(1-t_{n}\right)\left\|u-k_{n}\right\|}{t_{n}\left\|k_{n}-u\right\|} \\
& =1-\sqrt{\varepsilon_{n}} \longrightarrow 1 .
\end{aligned}
$$

Consider (3.25) for this choice of $k_{n}$. It yields

$$
\frac{r(u)-r\left(u+t_{n}\left(k_{n}-u\right)\right)}{t_{n}\left\|k_{n}-u\right\|} \leqq \frac{\left(-u^{*}, k_{n}-u\right)}{\left\|k_{n}-u\right\|}+\varepsilon \leqq\left\|u^{*}\right\|+\varepsilon
$$

for $n \geqq n_{0}$. Since the left-hand side converges to one we have the desired inequality $1-\varepsilon \leqq\left\|u^{*}\right\|$.

We show that in fact this method yields the following theorem. Here $m K=\left\{\left(x_{1}, x_{2}, \cdots, x_{m}\right): x_{1}=x_{2}=\cdots=x_{m} \in K\right\}$.

TheOREM 4.5. Let $\|\cdot\|_{i}, i=1,2, \cdots, m$ be equivalent norms with property $(\kappa)$. Let $K$ be relatively weakly compact and norm closed and consider

$$
\inf _{k \in K} \max _{1 \leqq i \leqq m}\left\|k-u_{i}\right\|_{i}=F\left(u_{1}, \cdots, u_{m}\right)
$$


where $u_{i}, i=1,2, \cdots, m$ are arbitrary points in $\prod_{i=1}^{m} V$. Then there is a dense $G_{\delta}$ set $T$ in $\prod_{i=1}^{m} V / K$ which has "simultaneous" nearest points in $K$.

Proof. Let $\|\cdot\|_{\infty}$ be defined on $\prod_{i=1}^{m} V$ by

$$
\left\|\left(v_{1}, \cdots, v_{m}\right)\right\|_{\infty}=\max _{i=1, \cdots, m}\left\|v_{i}\right\|_{i} .
$$

Set $\left(v_{1}, \cdots, v_{m}\right)=\bar{v}$. Then we may write

$$
F(\bar{v})=\inf _{m k \in m K}\|\bar{v}-m k\|_{\infty} \text {. }
$$

We may consider the sets $A_{\varepsilon}$, constructed before, for this problem. Then $T=\bigcap_{\varepsilon>0} A_{\varepsilon}$ is a dense $G_{\delta}$ set in $\prod_{i=1}^{m} V / m K$. Moreover, there is for each $u \in T$, by the construction of $T$, a minimizing sequence $m k_{n}$ in $m K$ with

$$
\left\|m k_{n}-u\right\|_{\infty} \longrightarrow\left\|m k_{0}-u\right\|_{\infty} ; m k_{n} \longrightarrow m k_{0} \text { (weakly). }
$$

Suppose that $\left\|m k_{0}-u\right\|_{\infty}=\left\|k_{0}-u_{1}\right\|_{1}$. There is some $i$ between 1 and $n$ with $\left\|k_{n}-u_{i}\right\|_{i} \rightarrow\left\|k_{0}-u_{1}\right\|_{1}$.

If $i=1$ it follows from property $(\kappa)$ that $k_{n}-u_{1}$ converges in $\|\cdot\|_{1}$ to $k_{0}-u_{1}$. Thus $k_{n}$ converges in each norm (by equivalence) to $k_{0} \in K$ and

$$
F\left(u_{1}, u_{2}, \cdots, u_{n}\right)=\max _{i=1, \cdots, n}\left\|k_{0}-u_{i}\right\|_{i} .
$$

If $i \neq 1,\left\|k_{0}-u_{i}\right\|_{i} \leqq\left\|k_{0}-u_{1}\right\|_{1}$ so that (since $\|\cdot\|_{i}$ is weakly lower semicontinuous) $\left\|k_{n}-u_{i}\right\|_{i}$ still converges to $\left\|k_{0}-u_{i}\right\|_{i}$. Again using property $(\kappa)$ for $\|\cdot\|_{i}$ completes the proof.

COROLlaRY 4.6. If $K$ is a norm-closed relatively weakly compact subset of a weakly compactly generated space $V$ with property $(\kappa)$, the set of points of $V / K$ with nearest points in $k$ contains $a$ dense $G_{\dot{o}}$ set.

This includes Lau's theorem in [13] as the reflexive case. Some condition like relative weak compactness is necessary since in the space $c_{0}$ with Day's locally uniformly convex norm, Cobzas [4] has provided a convex body for which the corollary fails. Similarly, some condition like $(\kappa)$ is necessary as Edelstein has exhibited a strictly convex renorming of $1_{2}$ for which it fails [9]. It would be interesting to know whether one can remove the strict convexity hypothesis of Theorem 4.1.

We note that the algorithm given in [7] for constructing dense nearest points in uniformly convex space can also be adjusted for 
simultaneous uniformly convex approximation. We also note that Theorem 4.5 holds because $\|\cdot\|_{\infty}$ has property $(\kappa)$ for directions in the diagonal.

\section{REFERENCES}

1. E. Asplund, Farthest points in locally uniformly rotund Banach spaces, Israel J. Math., 4 (1966), 213-216.

2. - Fréchet-differentiability of convex functions, Acta Math., 121 (1968), $31-47$.

3. J. Baranger and R. Teman, Nonconvex optimization problems depending on a parameter, SIAM J. Control, 13 (1974).

4. S. Cobzas, Antiproximinrl sets in Banach spaces, Math. Balkanica, 4 (1974), 79-82.

5. M. Day, Normed linear spaces, 3rd rev. ed., Ergebnisse der Math. und ihrer Grenzgebiete, Band 21, Springer-Verlag, Berlin and New York, 1973.

6. J. Diestel, Geometry of Banach Spaces, Lecture Notes in Math., 485, SpringerVerlag, Berlin and New York, 1975.

7. M. Edelstein, Farthest points of sets in uniformly convex Banach spaces, Israel

J. Math., 4 (1966), 171-176.

8. - On nearest points of sets in uniformly convex Banach spaces, J. London Math. Soc., 43 (1968), 375-377.

9. ——, Weakly proximinal sets, J. Approx. Th., 18 (1976), 1-8.

10. I. Ekeland, On the variational principle, J. Math. Anal. Appl., 47 (1974), 324-353.

11. I. Ekeland and G. Lebourg, Generic Fréchet-differentiability and Perturbed Optimation Problems in Banach spaces, Trans. Amer. Math. Soc., 224 (1976), 193-216.

12. K. Lau, On strongly exposing functionals, J. Aust. Math. Soc., 21 (Series A), (1976), 362-367.

13. - - A remark on strongly exposing functionals, Proc. Amer. Math. Soc.,

59 (1976),

14. - Almost Chebyshev subsets in reflexive Banach spaces, (to appear).

15. - Farthest points in weakly compact sets, Israel J. Math., 12 (1976), 162-174.

16. J. Rainwater, A theorem of Ekeland and Lebourg on Fréchet differentiability of convex functions on Banach spaces, (typed notes).

17. S. Steckin, Approximation Properties of Sets in Normed Linear Spaces, Rev. Math. Pures. Appl., 8 (1963), 5-18 (Russian).

18. F. Sullivan, Dentability, smoothability and stronger properties in Banach spaces, Indiana Math. J., (to appear).

19. S. Trojanski, On locally convex and differentiable norm in certain nonseparable Banach spaces, Studia Math., 37 (1971), 173-180.

Received March 30, 1978. Partially supported by NRC Account \#A4493.

DALHOUSIE UNIVERSITY

Halifax, N. S.

Canada B3H $4 \mathrm{H} 8$ 


\section{PACIFIC JOURNAL OF MATHEMATICS}

\section{EDITORS}

Donald BABBITT (Managing Editor)

University of California

Los Angeles, CA 90024

HUGo RossI

University of Utah

Salt Lake City, UT 84112

C. C. MOORE and ANDREW OGG

University of California

Berkeley, CA 94720

\section{J. DuGUNDJI}

Department of Mathematics

University of Southern California

Los Angeles, CA 90007

R. FINN and J. Milgram

Stanford University

Stanford, CA 94305

ASSOCIATE EDITORS
E. F. BECKENBACH
B. H. NeumanN
F. WOLF
K. YoSHIDA

\section{SUPPORTING INSTITUTIONS}

\author{
UNIVERSITY OF BRITISH COLUMBIA \\ CALIFORNIA INSTITUTE OF TECHNOLOGY \\ UNIVERSITY OF CALIFORNIA \\ MONTANA STATE UNIVERSITY \\ UNIVERSITY OF NEVADA, RENO \\ NEW MEXICO STATE UNIVERSITY \\ OREGON STATE UNIVERSITY \\ UNIVERSITY OF OREGON
}

\author{
UNIVERSITY OF SOUTHERN CALIFORNIA \\ STANFORD UNIVERSITY \\ UNIVERSITY OF HAWAII \\ UNIVERSITY OF TOKYO \\ UNIVERSITY OF UTAH \\ WASHINGTON STATE UNIVERSITY \\ UNIVERSITY OF WASHINGTON
}

The Supporting Institutions listed above contribute to the cost of publication of this Journal, but they are not owners or publishers and have no responsibility for its content or policies.

Mathematical papers intended for publication in the Pacific Journal of Mathematics should be in typed form or offset-reproduced, (not dittoed), double spaced with large margins. Please do not use built up fractions in the text of the manuscript. However, you may use them in the displayed equations. Underline Greek letters in red, German in green, and script in blue. The first paragraph or two must be capable of being used separately as a synopsis of the entire paper. Please propose a heading for the odd numbered pages of less than 35 characters. Manuscripts, in triplicate, may be sent to any one of the editors. Please classify according to the scheme of Math. Reviews, Index to Vol. 39. Supply name and address of author to whom proofs should be sent. All other communications should be addressed to the managing editor, or Elaine Barth, University of California, Los Angeles, California, 90024.

50 reprints to each author are provided free for each article, only if page charges have been substantially paid. Additional copies may be obtained at cost in multiples of 50 .

The Pacific Journal of Mathematics is issued monthly as of January 1966. Regular subscription rate: $\$ 84.00$ a year (6 Vols., 12 issues). Special rate: $\$ 42.00$ a year to individual members of supporting institutions.

Subscriptions, orders for numbers issued in the last three calendar years, and changes of address should be sent to Pacific Journal of Mathematics, P.O. Box 969, Carmel Valley, CA 93924, U.S.A. Older back numbers obtainable from Kraus Periodicals Co., Route 100, Millwood, NY 10546.

PUBLISHED BY PACIFIC JOURNAL OF MATHEMATICS, A NON-PROFIT CORPORATION

Printed at Kokusai Bunken Insatsusha (International Academic Printing Co., Ltd.). 8-8, 3-chome, Takadanobaba, Shinjuku-ku, Tokyo 160, Japan.

Copyright (C) 1979 by Pacific Journal of Mathematics Manufactured and first issued in Japan 


\section{Pacific Journal of Mathematics}

\section{Vol. 82 , No. 2 \\ February, 1979}

Krishnaswami Alladi and Paul Erdős, On the asymptotic behavior of large prime

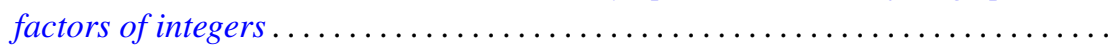

Alfred David Andrew, A remark on generalized Haar systems in $L_{p}$,

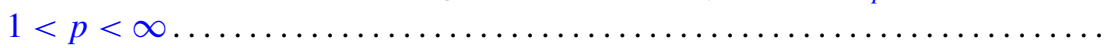

John M. Baker, A note on compact operators which attain their norm . . ........

Jonathan Borwein, Weak local supportability and applications to

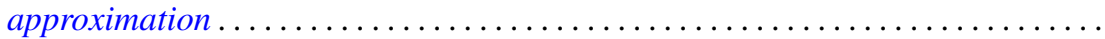

Tae Ho Choe and Young Soo Park, Wallman's type order compactification ........

Susanne Dierolf and Ulrich Schwanengel, Examples of locally compact

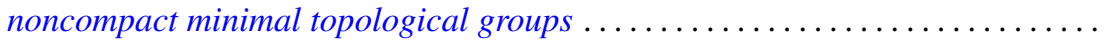

Michael Freedman, A converse to (Milnor-Kervaire theorem) $\times R$ etc. . . . . . . .

George Golightly, Graph-dense linear transformations ..................

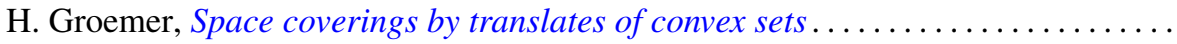

Rolf Wim Henrichs, Weak Frobenius reciprocity and compactness conditions in

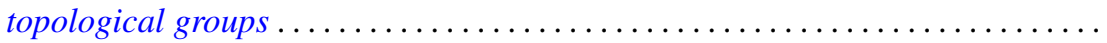

Horst Herrlich and George Edison Strecker, Semi-universal maps and universal

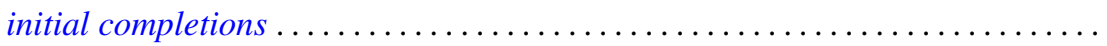

Sigmund Nyrop Hudson, On the topology and geometry of arcwise connected,

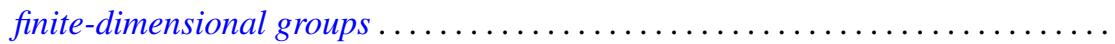

K. John and Václav E. Zizler, On extension of rotund norms. II .............

Russell Allan Johnson, Existence of a strong lifting commuting group of transformations. II.

Bjarni Jónsson and Ivan Rival, Lattice varieties covering the smallest nonmodular variety

Grigori Abramovich Kolesnik, On the order of Dirichlet L-functions .

Robert Allen Liebler and Jay Edward Yellen, In search of nonsolvable groups of

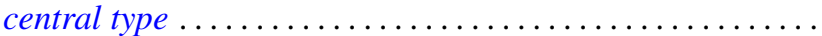

Wilfrido Martínez T. and Adalberto Garcia-Maynez Cervantes, Unicoherent plane Peano sets are $\sigma$-unicoherent ...

M. A. McKiernan, General Pexider equations. I. Existence of injective

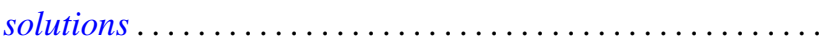

M. A. McKiernan, General Pexider equations. II. An application of the theory of webs.

Jan K. Pachl, Measures as functionals on uniformly continuous functions . .

Lee Albert Rubel, Convolution cut-down in some radical convolution algebras ...

Peter John Slater and William Yslas Vélez, Permutations of the positive integers

with restrictions on the sequence of differences. II . . .

Raymond Earl Smithson, A common fixed point theorem for nested spaces ....

Indulata Sukla, Generalization of a theorem of McFadden .... . . .

Jun-ichi Tanaka, A certain class of total variation measures of analytic measures.

Kalathoor Varadarajan, Modules with supplements .............. 